\title{
Neutron beam line for TOF measurements at the Spanish National Accelerator Lab (CNA)
}

\author{
Miguel Macías ${ }^{1,3, *}$, Begoña Fernández ${ }^{1,2, * *}$, and Javier Praena ${ }^{3, * * *}$ \\ ${ }^{1}$ Centro Nacional de Aceleradores (U. Sevilla, J. Andalucía, CSIC), Seville, Spain \\ ${ }^{2}$ Universidad de Sevilla, Seville, Spain \\ ${ }^{3}$ Universidad de Granada, Granada, Spain
}

\begin{abstract}
A few years ago, the Spanish National Accelerator Lab (CNA) developed the first accelerator-based neutron facility in Spain called HiSPANoS (HiSPAlis Neutron Source). The first applications of the line were related to integral measurements applied to nuclear astrophysics, dosimetry and single event effects produced by neutrons in electronic devices. The successful of HiSPANoS pushed the enhancement of the facility. In collaboration with the NEC® Company, two devices were designed for pulsing ion beams (Chopper) and for compressing in time (Buncher) the pulsed beams. The Chopper-Buncher system has been already installed and commissioned. Proton and deuteron beams are delivered with repetition rates from $62.5 \mathrm{kHz}$ to $2 \mathrm{MHz}$ and $1 \mathrm{~ns}$ pulse width. In addition, a new line of the 3 MV Tandem Pelletron accelerator was designed for neutron timeof-flight (TOF) experiments. Conventional devices and a dedicated Pick-Up for timing measurements form the new line. In order to check the performance of the whole TOF system, we have carried out the measurement of the neutron spectrum produced by ${ }^{7} \mathrm{Li}(\mathrm{p}, \mathrm{n}){ }^{7} \mathrm{Be}$ reaction at $\mathrm{E}_{p}=1912 \mathrm{keV}$. Such spectrum has been measured by the TOF technique few times and it can be considered a standard neutron field, in particular in nuclear astrophysics. The first result of such experiment performed at CNA is shown in some detail. The excellent performance of the accelerator, the Chopper-Buncher system and the acquisition system allow us to offer the TOF line at HiSPANoS-CNA to the neutron community.
\end{abstract}

\section{Introduction}

HiSPANoS is a facility which uses ion beams, provided by an accelerator, and nuclear reactions in order to generate neutrons. Accelerator-Based Neutron Sources (ABNS) play an important and complementary role to large neutron facilities. ABNS is relevant for neutron science in basic physics research, and play a significant role in a variety of applications, such as material science, education, medicine and is an industrial driver of the engineering. The facility has been set up around a NEC® (National Electrostatic Corp.) 9S DH-2 Tandem Pelletron accelerator, with a terminal potential of $3 \mathrm{MV}$ [1]. The ions

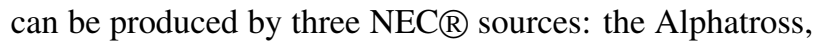
which generates ions from gases using radio frequency techniques; the Duoplasmatron, in which the ions are produced by bombarding the atoms of a chosen gas with electrons; and the SNICS II, a cesium sputtering source that produces ions from solid samples. The sources are connected to the $-30^{\circ}, 0^{\circ}$ and $+30^{\circ}$ input ports of an injection magnetic dipole respectively. Once the beam has been accelerated, it is analysed using a $90^{\circ}$ magnet. Then it can either pass directly to the $0^{\circ}$ line or can be deflected by a switcher magnet towards one of the other experimental lines placed at $\pm 15^{\circ}, \pm 30^{\circ}$ or $\pm 45^{\circ}$, as shown in Figure

\footnotetext{
*e-mail: mmacias4@us.es

**e-mail: bfernand@us.es

***e-mail: jpraena@ugr.es
}

1. The experimental neutron research takes place on the $+30^{\circ}$ one, which is called FNB line (Spanish acronym of Basic Nuclear Physics). All the fundamental parameters of the beam handling system (from the ion sources up to the switcher magnet) are remotely controlled and monitored from a console located in the control room outside the accelerator bunker [2].

Since then, some experiments in continuous wave (CW) have been carried out with different neutron production reactions. Briefly, the ${ }^{7} \mathrm{Li}(\mathrm{p}, \mathrm{n})^{7} \mathrm{Be}$ reaction has been used for producing different stellar neutron beams for nuclear astrophysics studies [3][4]. Also, this reaction has been used for producing thermal neutron beams for dosimetry studies in the conventional radiotherapy with photons [5][6] . The $\mathrm{D}(\mathrm{d}, \mathrm{n})^{3} \mathrm{He}$ reaction has been used for studies of soft errors rate in SRAM memories [7].

With the aim to employ the ${ }^{7} \operatorname{Li}(\mathrm{p}, \mathrm{n})$ and $\mathrm{D}(\mathrm{d}, \mathrm{n})$ reactions to generate the neutron pulses, a chopping/bunching system [8] was designed by NEC(R. It was designed to pulse proton and deuterium beams. The new system has been installed in the low energy zone at the 3 MV Tandem accelerator, upstream the Pelletron as shown in Figure 1. The success of this upgrade is endorsed by similar ones carried out in the second half of the last century $[9,10]$. A new experimental line has been designed and mounted after the $90^{\circ}$ magnet in the same direction as the accelerator tank, as shown in the scheme of Figure 1. This line 
is primarily focused on neutrons measures with the TimeOf-Flight technique (TOF).

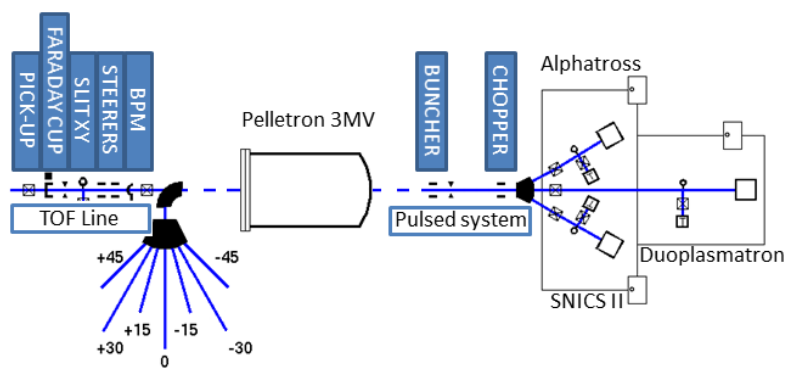

Figure 1. Schematic layout of the acceleration system and experimental beamlines after the upgrade, the new devices are highlighted in blue.

The TOF technique is applied to measure neutron energies [11-13]. A pulsed charge-particle beam generated by an accelerator comes on a target, and the produced neutrons (and/or $\gamma$ ) are observed in a detector at a distance $l$. The arrival time of the pulsed beam at the target (start signal) is obtained using a non-intercepting device such as a Pick-Up placed close to the target. The stop signal is provided by a detector. From the flight time $t$ measurement, the velocity $v=l / t$ of the particle can be obtained. If the mass $m$ of the particle is known, its energy can be determined with the equation (1).

$$
E=\frac{1}{2} m\left(\frac{l}{t}\right)^{2}
$$

The energy resolution is then determined with the equation (2).

$$
\frac{\Delta E}{E}=\left[\left(\frac{2 \Delta l}{l}\right)^{2}+\left(\frac{2 \Delta t}{t}\right)^{2}\right]^{1 / 2}
$$

where $\Delta l / l$ and $\Delta t / t$ are the fractional uncertainties in the measurement of the length of the flight path and of time respectively.

\section{HiSPANOS TOF}

\subsection{Pulsed system}

The pulsed system is composed by two units: a chopping and a bunching device. The goal of the pulsed system is to obtain a pulsed beam from a continuous beam. In Figure 2 a schematic view of the pulsed system installed on the Tandem accelerator is presented, the chopping elements and the bunching ones are highlighted in light and dark blue, respectively.

The beam chopper consists of a deflecting plate, mounted perpendicularly to the beam [14]. The plate is supplied with $650 \mathrm{Vdc}$ voltage by an electronic switch. During a period of one oscillation, the $d c$ voltage is always applied, thus deflecting the beam. Then, through pre-selection time windows, this voltage is cancelled. The beam is focused on an aperture and it is allowed to pass thus forming a pulse. The aperture is defined by a Slit XY

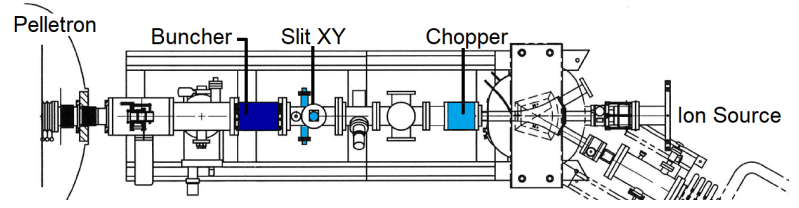

Figure 2. Schematic layout of the pulsed system installed on the Tandem accelerator, with the chopping (light blue) and the bunching (dark blue) elements.

located after the Chopper (see Figure 2). The distance between the Slit and the Chopper defines the drift distance. The frequency of the Chopper can be varied according to $2^{-n} \times 4 \mathrm{MHz}$, where $n=1,2, \ldots, 7$ in our case. The pulse width can be selected between 40 and 255 ns. Moreover, it is possible to set a delay between 0 and $255 \mathrm{~ns}$ to shift in time the pulse. In Table 1 a list of Chopper input parameters and their ranges is shown.

\begin{tabular}{cc}
\hline Chopper inputs & range \\
\hline Voltage $(\mathrm{V})$ & $0-650$ \\
Frequency $(\mathrm{MHz})$ & $2^{-n} \times 4, n=1,2, \ldots, 7$ \\
Width $(\mathrm{ns})$ & $40-255$ \\
Delay $(\mathrm{ns})$ & $0-255$ \\
\hline
\end{tabular}

Table 1. List of Chopper inputs that can be selected and their ranges.

Our bunching unit, composed by a pair of tubular electrodes, is mounted coaxially to the ion beam between the chopping unit and the Pelletron [15]. The electrodes are supplied with an $8 \mathrm{MHz}$ Radio-Frequency $(R F)$ voltage phase locked to the different frequencies of the chopping system. Both the entrance and the exit of the tubular bunching electrodes are used for the time compression of the pulsed beam. With the same polarity of the pulse, the $R F$-signal decreases the energy at the forefront of the pulse when it comes in the unit. Subsequently, the polarity is inverted increasing the energy at the pulse tail after its passage for it. The same system could be used for bunching protons and deuterons. In order to synchronise the Chopper pulse with the $R F$-signal, a delay to move in time the $R F$-signal between -90 and +90 degrees is available. The input pulses width must be shorter than $62.5 \mathrm{~ns}$ (a half period of the $R F$-signal). The maximum input frequency of the pulses is $8 \mathrm{MHz}$. The Buncher is designed to work with injection energy from the ion source of $58 \mathrm{keV}$ for proton and deuteron ions. In Table 2 a list of Buncher inputs is shown.

\subsection{Neutron TOF line}

Downstream the Pelletron, the ion pulsed beam enters in the TOF line. The TOF line is composed by: two gate valves located at both ends of the line, a pumping station, a Beam Profile Monitor, two magnetic Steerers, a Slit XY, a Faraday Cup, and a Pick-Up. These elements form the new dedicated experimental neutron line for the TOF technique, whose mission is to monitor and control the ion 


\begin{tabular}{cc}
\hline Buncher inputs & range \\
\hline Particles & $\mathrm{p} \mathrm{\& d}$ \\
Frequency $(\mathrm{MHz})$ & $2^{-n} \times 8, n=0,1, \ldots$ \\
Width (ns) & $0.00-62.50$ \\
Delay (degrees) & $-90.00-+90.00$ \\
Ion Energy (keV) & 58 \\
\hline
\end{tabular}

Table 2. List of Buncher inputs that can be selected and their ranges.

pulses. The Pick-Up is the most important device of the line, providing information about the frequency, width and timing (started signal) of pulses. A schematic view of the new experimental line is shown in Figure 3. This new line presents a compact size of $2 \mathrm{~m}$.

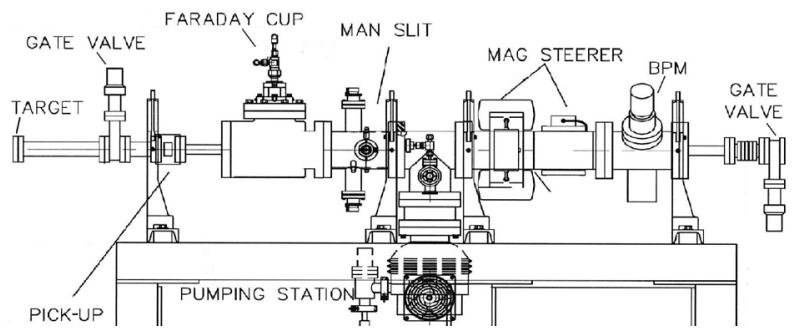

Figure 3. A schematic design of the TOF line composed by two gate valves located at both ends of line, a pumping station, a Beam Profile Monitor, two magnetic Steerers, a Slit XY, a Faraday Cup, and a Pick-Up (right to left beam direction). The new line presents a compact size of $2 \mathrm{~m}$.

\subsection{Pulsed system testing}

The goal of these tests is to determine the averagecurrent/frequency ratio $(\widehat{I} / f)$ and the peak-to-peak width $\left(W_{p p}\right)$ of the protons and deuterons pulsed beams. Both beams (proton and deuteron) were pulsed at each repetition rate, measuring constants average-current/frequency ratio (see Table 3). A peak-to-peak width of $1.6 \mathrm{~ns}$ was obtained with protons (see Figure 4) and 1.7 ns with deuterons (see Figure 5). The HiSPANoS facility was successfully tested, providing nanosecond pulsed beams of protons and deuterons.

\begin{tabular}{ccc}
\hline Ion pulsed beam & $\widehat{I} / f(\mathrm{nA} / \mathrm{kHz})$ & $W_{p p}(\mathrm{~ns})$ \\
\hline proton & 0.3 & 1.6 \\
deuteron & 0.1 & 1.7 \\
\hline
\end{tabular}

Table 3. List of average-current/frequency ratio $(\widehat{I} / f)$ and the peak-to-peak width $\left(W_{p p}\right)$ of the protons and deuterons pulsed beams measured during the tests.

\section{Commissioning}

In 1988, Ratynski and Käppeler published the MACS of

${ }^{197} \mathrm{Au}(\mathrm{n}, \gamma)$ at $\mathrm{kT}=30 \mathrm{keV}$ with a neutron field produced

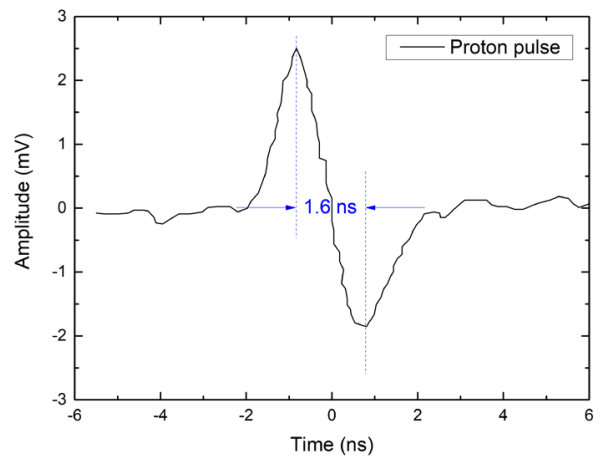

Figure 4. Signal induced into the Pick-Up by a proton pulse. It presents a peak-to-peak width of $1.6 \mathrm{~ns}$.

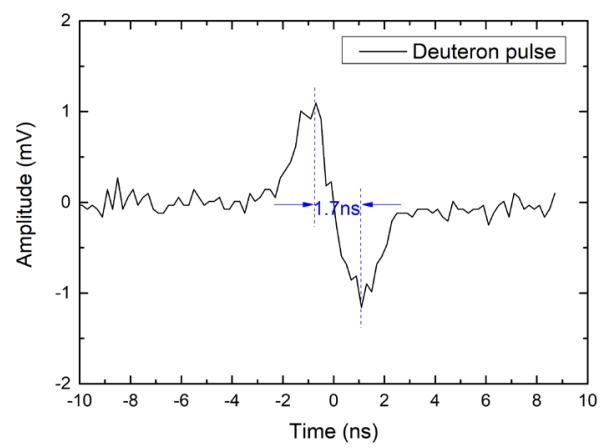

Figure 5. Signal induced to the Pick-Up by a deuteron pulse. It presents a peak-to-peak width of $1.7 \mathrm{~ns}$.

by protons at $1912 \mathrm{keV}$ impinging on a thick lithium target. Such neutron field, measured with the TOF technique, has been very important in nuclear astrophysics because it resembles in good approximation the stellar field in important nucleosynthesis scenarios [11]. In the last years, there have been some TOF experimental works for establishing the R\&K field as a neutron standard [12]. The consistent experimental results of the neutron field obtained by $\mathrm{R} \& \mathrm{~K}$ [11], Lederer et al. [12] and Feinberg et al. [13] have shown the robustness of the method. Therefore, the measurement of the well-known neutron field of R\&K is an excellent commissioning of the novel TOF system developed at CNA for neutrons. The accelerator was operated in pulsed wave at $500 \mathrm{kHz}$. A stable proton pulses average current of $100 \mathrm{nA}$ was produced. The experimental set-up consisted of a target assembly accomplished to the beam pipe of the TOF line and a ${ }^{6} \mathrm{Li}$-glass neutron detector purchased from Scionix Ltd. [16]. The target assembly consisted of an aluminium cylinder $(4.2 \mathrm{~cm}$ diameter and $15 \mathrm{~cm}$ length) and a copper backing for the lithium target. The only materials that neutron beam found from the source to the detectors were the copper backing and the lithium. The copper backing holding the lithium target was cooled by a forced air flow on the external side. The pressure was $\approx 10^{-6}$ mbar during the whole experiment. The ${ }^{6} \mathrm{Li}$-glass detector was located at the same height of the lithium target. The detector $(5.08 \mathrm{~cm}$ diameter and $2.54 \mathrm{~cm}$ thick) recorded the signals at $0^{\circ}$ (with 
respect to the beam direction) and with a flight path of $50 \mathrm{~cm}$. The Pick-Up signal (started one) and the detector signals (stopped one) were processed by a Digitizer Desktop model DT5730 of CAEN, a 8 channels 14 bit at $500 \mathrm{Ms} / \mathrm{s}$ FLASH ADC Waveform Digitizer [17]. In Figure 6 a $2 \mathrm{D}$ scatter plot of the TOF versus the charge of the detector signals is shown. The $\gamma$-rays appear as a vertical line in time close to $100 \mathrm{~ns}$. The neutrons appear in the horizontal band from $200 \mathrm{~ns}$ with a drop shape. It can be noticed the excellent discrimination between $\gamma$-rays and neutrons during the experiment. The neutron scattering component of the background is clearly identified between the $\gamma$-flash and the onset of the neutron drop and it is uncorrelated with time, as can be seen before the neutron drop. Our result will be directly compared with the pre-

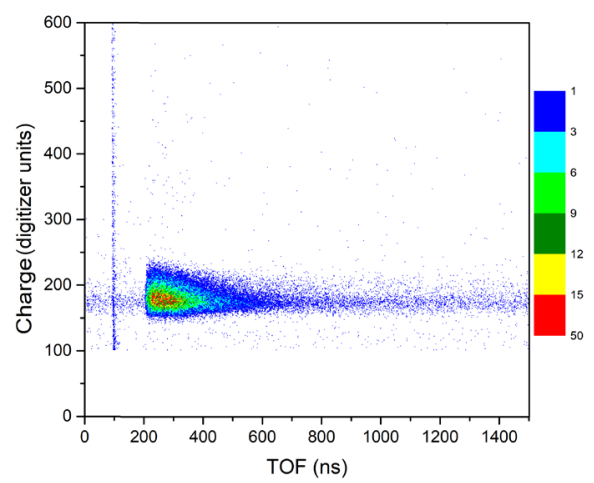

Figure 6. 2D scatter plots of the TOF versus the charge of the detector signals. The $\gamma$-rays appear as a vertical line and the neutrons appear in the horizontal band with a drop shape.

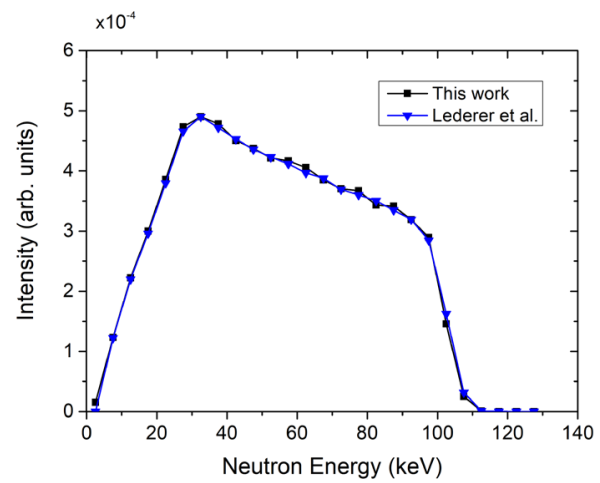

Figure 7. Experimental neutron spectrum of this work at $0^{\circ}$, $50 \mathrm{~cm}$ flight path, and $500 \mathrm{kHz}$ (black line+squares) compared to Lederer et al. [12] (blue line+triangles) in bins of $5 \mathrm{keV}$. Statistical uncertainties are smaller than the black square size.

vious measurements of $\mathrm{R} \& \mathrm{~K}$ field [11]. The comparison regards to the neutron spectrum obtained at $0^{\circ}$. We will compare to Lederer et al. [12] because the data are avail- able on-line and agree with the results of R\&K (see EXFOR data base [18]). Figure 7 shows our result (black line) of the energy spectrum at $0^{\circ}$ in bins of $5 \mathrm{keV}$ obtained at $50 \mathrm{~cm}$ (flight paths) and $500 \mathrm{kHz}$ (repetition rate) in comparison with Lederer et al. (blue line) re-binned to $5 \mathrm{keV}$ for a better comparison. Our energy spectrum is obtained after a flat background subtraction in the TOF histogram, a TOF to energy conversion, and finally applying the corresponding normalisation to compare the spectra. The good agreement between the results obtained with the Lederer et al. data [18] demonstrates the good performance of the whole TOF system for neutrons developed at CNA.

\section{Acknowledgements}

The authors acknowledge financial support from the Spanish MINECO funds (FPA2013-47327-C2-1-R, BES-2014-068808, and FPA2016-77689-C2-1-R), AECC (PS16163811PORR), Spanish Fundación ACS, Capitán Antonio, and FEDER Andalucía (US-1261006).

\section{References}

[1] NEC@, http://www.pelletron.com/index.html (2019)

[2] J. García-López et al., Nucl. Instr. Meth. Phys. Res. B 161-163, 1137 (2000)

[3] J. Praena et al., Nucl. Instr. Meth. Phys. Res. A 727, 1 (2013)

[4] J. Praena et al., Nucl. Data Sheets 120, 205 (2014)

[5] J. Praena et al., Radiother. Oncol. 115, S735 (2015), 3rd ESTRO Forum 24-28 April 2015, Barcelona, Spain

[6] L. Irazola et al., Appl. Radiat. Isotopes 107, 330 (2016)

[7] D. Malagón et al., Microelectron. Reliab. 78, 38 (2017)

[8] J. Anderson, D. Swann, Nucl. Instr. Meth. 30, 1 (1964)

[9] C. Moak et al., Rev. Sci. Instrum. 35, 672 (1964)

[10] A. Crametz et al., Nucl. Instr. Meth. Phys. Res. A 242, 179 (1986)

[11] W. Ratynski, F. Kappeler, Phys. Rev. C 37, 595 (1988)

[12] C. Lederer et al., Phys. Rev. C 85, 055809(8) (2012)

[13] G. Feinberg et al., Phys. Rev. C 85, 055810(12) (2012)

[14] T. Fowler, W. Good, Nucl. Instr. Meth. 7, 245 (1960)

[15] P. Tykesson, T. Wiedling, Nucl. Instr. Meth. 77, 277 (1970)

[16] Scionix Ltd., https://scionix.nl/ (2019)

[17] Caenß, https://www.caen.it/ (2019)

[18] EXFOR database (2019-05-29), https://www-nds. iaea.org/exfor/exfor.htm (2019) 